\title{
Executing Instructions in Situated Collaborative Interactions
}

\author{
Alane Suhr \\ Cornell University \\ suhrecs. cornell. edu
}

\author{
Claudia Yan \\ IBM \\ claudiab.yan@gmail.com
}

\author{
Jacob Schluger* \\ Cornell University \\ jes543@cornell. edu
}

\author{
Stanley Yu* \\ Columbia University \\ stanley.yuecolumbia.edu
}

\author{
Hadi Khader** \\ Intel \\ hadi.kh.khader@gmail.com
}

\section{Marwa Mouallem** IBM}

marwamoual lem@gmail.com

\author{
Iris Zhang \\ Facebook \\ iriszefb.com
}

Yoav Artzi

Cornell University

yoav@cs. cornell. edu

\begin{abstract}
We study a collaborative scenario where a user not only instructs a system to complete tasks, but also acts alongside it. This allows the user to adapt to the system abilities by changing their language or deciding to simply accomplish some tasks themselves, and requires the system to effectively recover from errors as the user strategically assigns it new goals. We build a game environment to study this scenario, and learn to map user instructions to system actions. We introduce a learning approach focused on recovery from cascading errors between instructions, and modeling methods to explicitly reason about instructions with multiple goals. We evaluate with a new evaluation protocol using recorded interactions and online games with human users, and observe how users adapt to the system abilities.
\end{abstract}

\section{Introduction}

Sequential instruction scenarios commonly assume only the system performs actions, and therefore only its behavior influences the world state. This ignores the collaborative potential of such interactive scenarios and the challenges it introduces. When the user acts in the world as well, they can adapt to the system abilities not only by adopting simpler language, but also by deciding to accomplish tasks themselves. The system must then recover from errors as new instructions arrive and be robust to changes in the environment that are not a result of its own actions.

In this paper, we introduce CEREALBAR, a collaborative game with natural language instruction, and design modeling, learning, and evaluation methods for the problem of sequential instruction following in collaborative interactions. In CEREALBAR, two agents, a leader and a follower,

\footnotetext{
${ }^{*},{ }^{* *}$ : Equal contribution. All work done at Cornell.
}

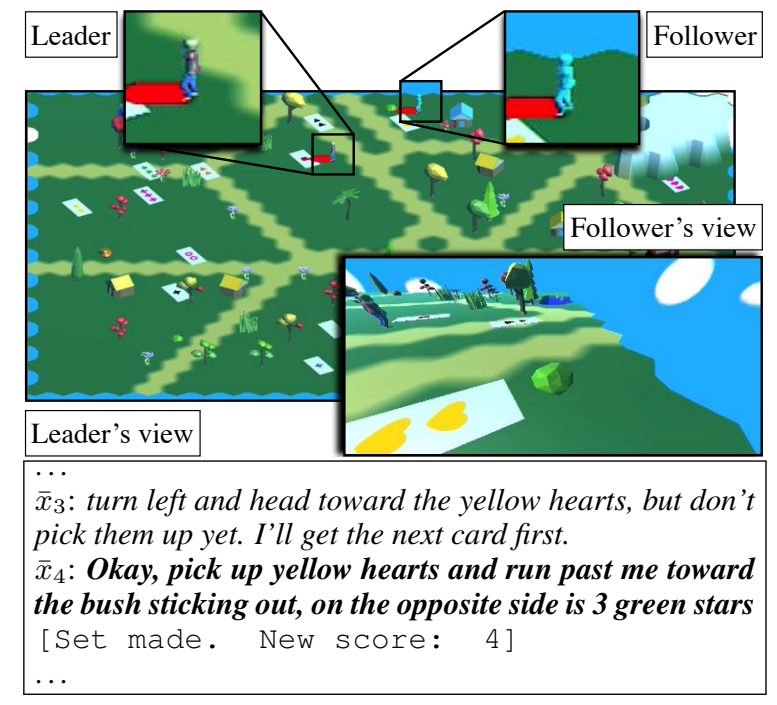

Figure 1: A snapshot from an interaction in CEREALBAR. The current instruction is in bold. The large image shows the entire environment. This overhead view is available only to the leader. The follower sees a firstperson view only (bottom right). The zoom boxes (top) show the leader and follower.

move in a $3 \mathrm{D}$ environment and collect valid sets of cards to earn points. A valid set is a set of three cards with distinct color, shape, and count. The game is turn-based, and only one player can act in each turn. In addition to collecting cards, the leader sends natural language instructions to the follower. The follower's role is to execute these instructions. Figure 1 shows a snapshot from the game where the leader plans to pick up a nearby card (red square) and delegates to the follower two cards, one close and the other much further away. Before that, the leader planned ahead and asked the follower to move in preparation for the next set. The agents have different skills to incentivize collaboration. The follower has more moves per turn, but can only see from first-person view, while the leader observes the entire environment but has fewer moves. This makes natural language inter- 
action key to success. We address the problem of mapping the leader instructions to follower actions. In addition to the collaborative challenges, this requires grounding natural language to resolve spatial relations and references to objects, reason about dependencies on the interaction history, react to the changing environment as cards appear and disappear, and generate actions.

CEREALBAR requires reasoning about the changing environment (e.g., when selecting cards) and instructions with multiple goals (e.g., selecting multiple cards). We build on the Visitation Prediction Network model (VPN; Blukis et al., 2018b), which casts planning as mapping instructions to the probability of visiting positions in the environment. Our new model generalizes the planning space of VPN to reason about intermediate goals and obstacles, and includes recurrent action generation for trajectories with multiple goals.

We collect 1,202 human-to-human games for training and evaluation. While our model could be trained from these recorded games only, it would often fail when an instruction would start at the wrong position because of an error in following the previous one. We design a learning algorithm that dynamically augments the data with examples that require recovering from such errors, and train our model to distinguish such recovery reasoning from regular instruction execution.

Evaluation with recorded games poses additional challenges. As agent errors lead to unexpected states, later instructions become invalid. Because measuring task completion from such states is meaningless, we propose cascaded evaluation, a new evaluation protocol that starts the agent at different points in the interaction and measures how much of the remaining instructions it can complete. In contrast to executing complete sequences or single instructions, this method allows to evaluate all instructions while still measuring the effects of error propagation.

We evaluate using both static recorded games and live interaction with human players. Our human evaluation shows users adapt to the system and use the agent effectively, scoring on average 6.2 points, compared to 12.7 for human players. Our data, code, and demo videos are available at lil.nlp.cornell.edu/cerealbar/.

\section{Setup and Technical Overview}

We consider a setup where two agents, a leader and a follower, collaborate. Both execute actions in a shared environment. The leader, additionally, instructs the follower using natural language. The leader goal is to maximize the task reward, and the follower goal is to execute leader instructions. We consider a turn-based version, where at each turn only one agent acts. We instantiate this scenario in CerealBAR, a navigation card game (Figure 1), where a leader and follower move in an environment selecting cards to complete sets. ${ }^{1}$

Cerealbar Overview The objective of CEREALBAR is to earn points by selecting valid sets of cards. A valid set has three cards with distinct color, shape, and count. When the only cards selected in the world form a valid set, the players receive a point, the selected cards disappear, three new cards are added randomly, and the number of remaining turns increases. The increase in turns decays for each set completed. An agent stepping on a card flips its selection status. The players form sets together. The follower has more steps per turn than the leader. This makes using the follower critical for success. The follower only sees a first-person view of the environment, preventing them from planning themselves, and requiring instructions to be sensible from the follower's perspective. The leader chooses the next target set, plans which of the two players should get which card, and instructs the follower. The follower can not respond to the leader, and should not plan themselves, or risk sabotaging the leader's plan, wasting moves and lowering their potential score. Followers mark an instruction as finished before observing the next one. This provides alignment between instructions and follower actions. In contrast to the original setup that we use for data collection, in our model (Section 4), we assume the follower has full observability, leaving the challenge of partial observability for future work. Appendix A provides further game design details.

Problem Setup We distinguish between the world state and the interaction state. Let $\mathcal{S}$ be the set of all world states, $\Gamma$ be the set of all interaction states, and $\mathcal{X}$ be the set of all natural language instructions. A world state $s \in \mathcal{S}$ describes the current environment. In CEREALBAR, the world state describes the spatial environment, the location of cards, whether they are selected or not, and the location of the agents. An interaction state $\gamma \in \Gamma$ is a tuple $\langle\bar{Q}, \alpha, \psi\rangle$. The first-in-first-out

\footnotetext{
${ }^{1}$ The name CEREALBAR does not carry special meaning. It was given to the project early on, and we came to like it. Our game is inspired by the card game Set.
} 
queue $\bar{Q}=\left[\bar{x}_{q}, \ldots, \bar{x}_{q^{\prime}}\right]$ contains the instructions $\bar{x}_{i} \in \mathcal{X}$ available to execute. The current instruction is the left-most instruction $\bar{x}_{q}$. The current turn-taker $\alpha \in\{$ Leader, Follower $\}$ indicates the agent currently executing actions, and $\psi \in \mathbb{N}_{\geq 0}$ is the number of steps remaining in the current turn.

At each time step, the current turn-taker agent takes an action. An action may be the leader issuing an instruction, or either agent performing an action in the environment. Let $\mathcal{A}=\mathcal{A}_{w} \cup$ $\{$ DONE $\} \cup \mathcal{X}$ be the set of all actions. The set $\mathcal{A}_{w}$ includes the actions available to the agents in the environment. In CEREALBAR, this includes moving forward or backward, and turning left or right. Moving onto a card flips it selection status. DONE indicates completing the current instruction for the follower or ending the turn for the leader. An instruction action $a=\bar{x} \in \mathcal{X}$ can only be taken by the leader and adds the instruction $\bar{x}$ to the queue $\bar{Q}$. The effect of each action is determined by the transition function $\mathcal{T}: \mathcal{S} \times \Gamma \times \mathcal{A} \rightarrow \mathcal{S} \times \Gamma$, which is formally defined in Appendix B. Only world actions $a \in \mathcal{A}_{w}$ decrease the remaining steps $\psi$.

The goal of the leader is to maximize the total reward of the interaction. An interaction $\bar{I}=$ $\left\langle\left(s_{1}, \gamma_{1}, a_{1}\right), \ldots,\left(s_{|\bar{I}|}, \gamma_{|\bar{I}|}, a_{|\bar{I}|}\right)\right\rangle$ is a sequence of state-action tuples, where $\mathcal{T}\left(s_{i}, \gamma_{i}, a_{i}\right)=$ $\left(s_{i+1}, \gamma_{i+1}\right)$. The reward function $R: \mathcal{S} \times \mathcal{A} \rightarrow \mathbb{R}$ assigns a numerical reward to a world state and an action. The total reward of an interaction $\bar{I}$ is $\sum_{i=0}^{|\bar{I}|} R\left(s_{i}, a_{i}\right)$. In CEREALBAR, the agents receive a reward of 1 when a valid set is selected.

Task Our goal is to learn a follower policy to execute the leader instructions. At time $t$, given the current world and interaction states $s_{t}$ and $\gamma_{t}$, and the interaction so far $\bar{I}_{<t}$, the follower policy $\pi\left(s_{t}, \gamma_{t}, \bar{I}_{<t}\right)$ predicts the next action $a_{t}$.

Model We decompose the follower policy $\pi\left(s_{t}, \gamma_{t}, \bar{I}_{<t}\right)$ to predicting a set of distributions over positions in the environment, including positions to visit, intermediate goals (e.g., cards to select), positions to avoid (e.g., cards not to touch), and positions that are not passable. These distribution are used in a second stage to generate a sequence of actions. Section 4 describes the model.

Learning We assume access to a set of $N$ recorded interactions $\left\{\bar{I}^{(i)}\right\}_{i=1}^{N}$, and create examples where each instruction is paired with a sequence of state-action tuples. We maximize the action-level cross entropy objective, and use two auxiliary objectives (Section 5). We first train each stage of the model separately, and then fine-tune them jointly. During fine-turning, we continuously generate additional examples using model failures. These examples help the agent to learn how to recover from errors in prior instructions.

Evaluation We measure correct execution of instructions and the overall game reward. We assume access to a test set of $M$ recorded interactions $\left\{\bar{I}^{(i)}\right\}_{i=1}^{M}$. We measure instruction-level and interaction-level performance, and develop cascaded evaluation, an evaluation protocol that provides a more graded measure than treating each interaction as a single example, while still accounting for error propagation (Section 6). Finally, we conduct online evaluation with human leaders.

\section{Related Work}

Goal-driven natural language interactions have been studied in various scenarios, including dialogue where only one side acts in the world (Anderson et al., 1991; Williams et al., 2013; Vlachos and Clark, 2014; de Vries et al., 2018; Kim et al., 2019; Hu et al., 2019), coordination for agreed selection of an object (He et al., 2017; Udagawa and Aizawa, 2019), and negotiation (Lewis et al., 2017; He et al., 2018). We focus on collaborative interactions where both the user and the system perform sequences of actions in the same environment. This allows the user to adapt to the language understanding ability of the system and balance between delegating goals to it and accomplishing them themselves. For example, a user may decide to complete a short but hard-to-describe task and delegate to the system a long but easy-to-describe one. In prior work, in contrast, recovery is limited to users paraphrasing their requests. The Cards corpus (Djalali et al., 2011, 2012; Potts, 2012) was used for linguistic analysis of collaborative bi-directional language interaction. The structure of collaborative interactions was also studied using Wizard-of-Oz studies (Lochbaum, 1998; Sidner et al., 2000; Koulouri and Lauria, 2009). In contrast, we focus on building agents that follow instructions. Ilinykh et al. (2019) present a corpus for the related task of natural language coordination in navigation. Collaboration has also been studied for emergent communication (e.g., Andreas et al., 2017; Evtimova et al., 2017).

Understanding sequences of natural language utterances has been addressed using semantic parsing (e.g., Miller et al., 1996; MacMahon et al., 2006; Chen and Mooney, 2011; Artzi and Zettlemoyer, 2013; Artzi et al., 2014; Long et al., 2016; 
Iyyer et al., 2017; Suhr et al., 2018; Arkin et al., 2017; Broad et al., 2017). Interactions were also used for semantic parser induction (Artzi and Zettlemoyer, 2011; Thomason et al., 2015; Wang et al., 2016). These methods require hand-crafted symbolic meaning representation, while we use low-level actions (Suhr and Artzi, 2018). The interactions in our environment interleave actions of both agents with leader utterances, an aspect not addressed by these methods. Executing single instructions has been widely studied (e.g., Tellex et al., 2011; Duvallet et al., 2013; Misra et al., 2017, 2018; Anderson et al., 2018; Blukis et al., 2018a,b; Chen et al., 2019). The distinction we make between actions specified in the instruction and implicit recovery actions is similar to how Artzi and Zettlemoyer (2013) use implicit actions for single instructions. Finally, our model is based on the VPN model of Blukis et al. (2018b). While we assume full observability, their original work did not. This indicates that our model is likely to generalize well to partially observable scenarios.

\section{Model}

We use a two-stage model for the follower policy $\pi\left(s_{t}, \gamma_{t}, \bar{I}_{<t}\right)$, where $s_{t}$ is a world state, $\gamma_{t}$ is an interaction state, and $\bar{I}_{<t}$ is the interaction history. The instruction $\bar{x}$ that is the first in the queue $\bar{Q}_{t}$, which is part of $\gamma_{t}$, is the currently executed instruction. In our model, we assume the follower observes the entire environment. First, we map $\bar{x}$ and $s_{t}$ to distributions over locations in the environment, including what locations to visit and what are the goals. These distributions are considered as an execution plan, and are used to generate a sequence of actions in the second stage. The distribution can also be used to easily easily visualize the agent plan. The first stage is used when starting a new instruction, and the predicted distributions are re-used for all actions for that instruction. Figure 2 illustrates the architecture and the distributions visualization. The two-stage approach was introduced by Blukis et al. (2018b). We generalize its planning space and add a recurrent action generator for execution.

Input Representation The inputs to the first stage are the instruction $\bar{x}$ and the world state $s_{t}$. We generate feature maps for both. We use a learned embedding function $\phi^{\mathcal{X}}$ and a bi-directional recurrent neural network (RNN; Elman, 1990) with a long short-term memory cell (LSTM; Hochreiter and Schmidhuber, 1997)
$\mathrm{RNN}^{\mathcal{X}}$ to map $\bar{x}$ to a vector $\overline{\mathbf{x}}$. The world state $s_{t}$ is a $3 \mathrm{D}$ tensor that encodes the properties of each position. The dimensions of $s_{t}$ are $P \times W \times H$, where $P$ is the number of properties, and $W$ and $H$ are the environment width and height. Each of the $W \times H$ positions is represented in $s_{t}$ as a binary vector of length $P$. For example, a position with a red hut will have 1's for the red and hut dimensions and 0's for all other dimensions. We map the world state to a tensor feature map $\mathbf{F}_{0}$ by embedding $s_{t}$ and processing it using the text representation $\overline{\mathbf{x}}$. We use a learned embedding function $\phi^{\mathcal{S}}$ to map each position vector to a dense embedding of size $N_{s}$ by summing embeddings of each of the position's properties. The embeddings are combined to a tensor $\mathbf{S}$ of dimension $N_{s} \times W \times H$ representing a featurized global view of the environment. We create a text-conditioned state representation by creating a kernel $\mathbf{K}_{s}$ and convolving with it over $\mathbf{S}$. We use a linear transformation to create $\mathbf{K}_{s}=\mathbf{W}_{s} \overline{\mathbf{x}}+\mathbf{b}_{s}$, where $\mathbf{W}_{s}$ and $\mathbf{b}_{s}$ are learned weights. We reshape $\mathbf{K}_{s}$ to a $1 \times 1$ convolution kernel with $N_{s^{\prime}}$ output channels, and compute $\mathbf{S}^{\prime}=\mathbf{S} * \mathbf{K}_{s}$. We concatenate $\mathbf{S}$ and $\mathbf{S}^{\prime}$ along the channel dimension and rotate and center so the follower position is at center pixel to generate $\mathbf{F}_{0}{ }^{2}$ Stage 1: Plan Prediction We treat plan generation as predicting distributions over positions $\rho$ in the environment. There are $W \times H$ possible positions. We predict four distributions: (a) $p\left(\rho \mid s_{t}, \bar{x}\right)$, the probability of visiting $\rho$ while executing the instruction $\bar{x}$; (b) $p\left(\mathrm{GOAL}=1 \mid \rho, s_{t}, \bar{x}\right)$, the binary probability that $\rho$ is a goal (i.e., GOAL $=1$ when containing a card to select); (c) $p\left(\right.$ AVOID $\left.=1 \mid \rho, s_{t}, \bar{x}\right)$, the binary probability that the agent must not pass in $\rho$ (i.e., AVOID $=1$ when it contains a card that should not be touched); and (d) $p\left(\mathrm{NOPASS}=1 \mid \rho, s_{t}, \bar{x}\right)$, the binary probability the agent cannot pass in $\rho$ (i.e., NOPASS $=1$ when it contains another object).

We use LingUNET (Misra et al., 2018) to predict the distributions. The inputs to LINGUNET are the instruction embedding $\overline{\mathbf{x}}$ and featurized world state $\mathbf{F}_{0}$, which is relative to the agent's frame of reference. The output are four matrices, each of dimension $W \times H$ corresponding to the environment. LINGUNET is formally defined in Misra et al. (2018) and Appendix D. Roughly

\footnotetext{
${ }^{2}$ Appendix D describes the relationship between the environment representations and the agent's initial and current orientation.
} 


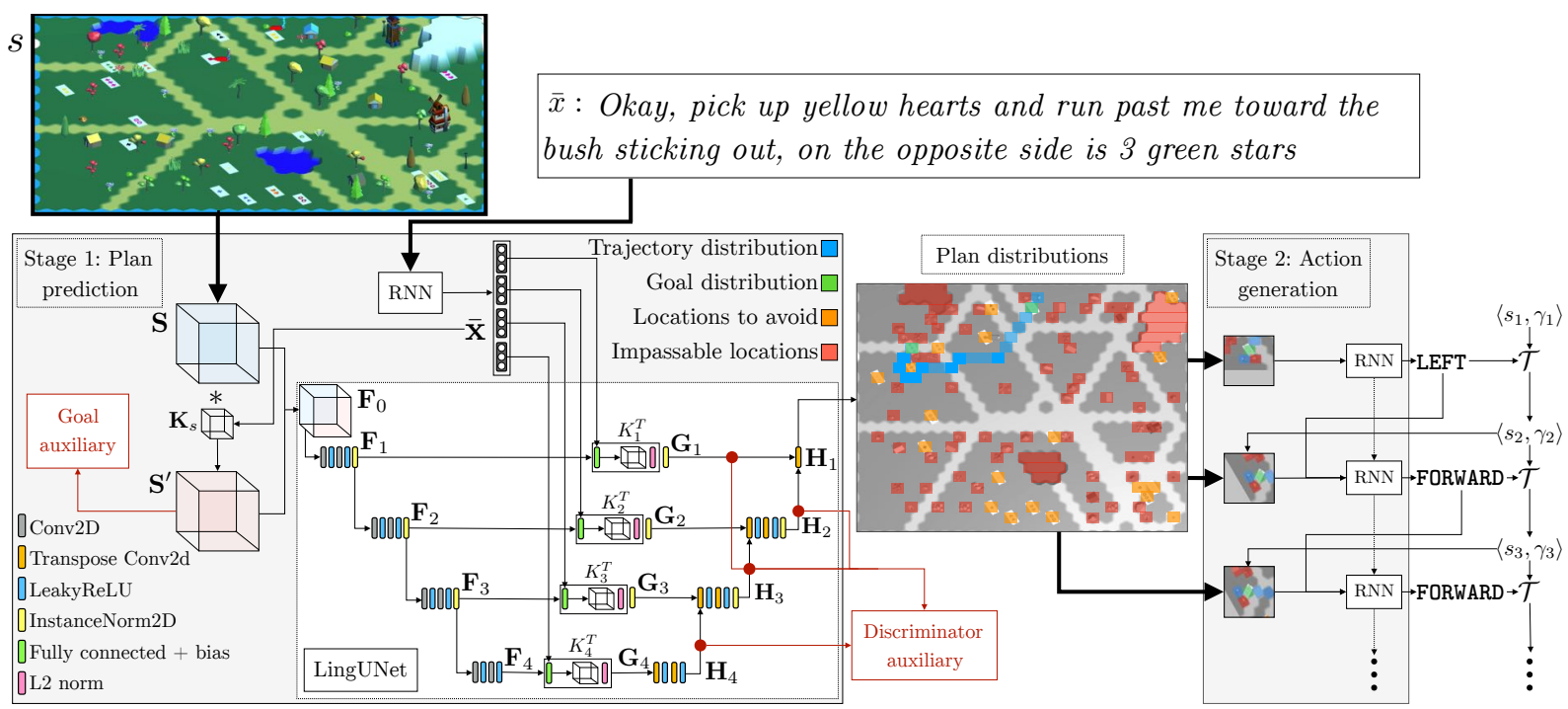

Figure 2: Illustration of the model architecture. Given the instruction $\bar{x}$ and the world state $s$, we compute $\mathbf{F}_{0}$ from the embeddings of the instruction $\overline{\mathbf{x}}$ and environment $\mathbf{S}$. We use LINGUNET to predict four distributions, which are visualized over the map (grayscaled to emphasize the distributions). We show three action generation steps. Each step receives the map cropped around the agent and the previous action, and outputs the next action.

speaking, LINGUNET reasons about the environment representation $\mathbf{F}_{0}$ at $L$ levels. First, $\mathbf{F}_{0}$ is used to generate feature maps of decreasing size $\mathbf{F}_{j}, j=1 \ldots L$ using a series of convolutions. We create convolution kernels from the instruction representation $\overline{\mathbf{x}}$, and apply them to the feature maps $\mathbf{F}_{j}$ to generate text-conditioned feature maps $\mathbf{G}_{j}$. Finally, feature maps of increasing size $\mathbf{H}_{j}$ are generated using a series of $L$ deconvolutions. The last deconvolution generates a tensor of size $4 \times W \times H$ with a channel for each of the four distributions. We use a softmax over one channel to compute $p\left(\rho \mid s_{t}, \bar{x}\right)$. Because the other distributions are binary, we use a sigmoid on each value independently for the other channels. When computing $p\left(\mathrm{GOAL}=1 \mid \rho, s_{t}, \bar{x}\right)$ and $p\left(\right.$ AVOID $\left.=1 \mid \rho, s_{t}, \bar{x}\right)$ we mask positions without objects that can be changed (i.e., positions without cards) to assign them zero probability.

Stage 2: Action Generation We use the four distributions to generate a sequence of actions. We concatenate the distributions channel-wise to a tensor $\mathbf{P} \in \mathbb{R}^{4 \times W \times H}$. We use a forward LSTM RNN to predict a sequence of actions. At each prediction step $t$, we rotate, transform, and crop $\mathbf{P}$ to generate the egocentric tensor $\mathbf{P}_{t}^{\prime} \in \mathbb{R}^{N^{\prime} \times C \times C}$, where the agent is always at the center and facing in the same direction, such that $\mathbf{P}_{t}^{\prime}$ is relative to the agent's current frame of reference. The input to the action generation $\mathrm{RNN}$ at time $t$ is:

$$
\begin{aligned}
& \mathbf{p}_{t}^{\prime}=\operatorname{vec}\left(\operatorname{Norm}\left(\operatorname{ReLU}\left(\operatorname{CNN}^{P}\left(\mathbf{P}_{t}^{\prime}\right)\right)\right)\right) \\
& \mathbf{p}_{t}=\operatorname{ReLU}\left(\mathbf{W}_{2}^{P}\left[\mathbf{p}_{t}^{\prime} ; \operatorname{ReLU}\left(\mathbf{W}_{1}^{P} \mathbf{p}_{t}^{\prime}+\mathbf{b}_{1}^{P}\right)\right]+\mathbf{b}_{2}^{P}\right),
\end{aligned}
$$

where $\mathrm{CNN}^{P}$ is a convolutional layer, RELU is a non-linearity, NORM is instance normalization (Ulyanov et al., 2017), and $\mathbf{W}_{1}^{P}, \mathbf{W}_{2}^{P}, \mathbf{b}_{1}^{P}$, $\mathbf{b}_{2}^{P}$ are learned weights. The action probability is:

$$
\begin{aligned}
\mathbf{h}_{t} & =\operatorname{RNN}^{\mathcal{A}}\left(\mathbf{h}_{t-1},\left[\phi^{\mathcal{A}}\left(a_{t-1}\right) ; \mathbf{p}_{t}\right]\right) \\
p(a) & \propto \exp \left(\mathbf{W}^{\mathcal{A}}\left[\mathbf{h}_{t} ; \mathbf{p}_{t}\right]+\mathbf{b}^{\mathcal{A}}\right),
\end{aligned}
$$

where $\mathrm{RNN}^{\mathcal{A}}$ is an LSTM RNN, $\phi^{\mathcal{A}}$ is a learned action embedding function, $a_{0}$ is a special START action, and $\mathbf{W}^{\mathcal{A}}$ and $\mathbf{b}^{\mathcal{A}}$ are learned. During inference, we assign zero probabilities to actions $a$ when $\mathcal{T}_{w}\left(s_{t}, a\right)$ is invalid (Appendix B), for example when an agent would move into an obstacle.

\section{Learning}

We assume access to a set of $N$ recorded interactions $\left\{\bar{I}^{(i)}\right\}_{i=1}^{N}$. We generate instruction-level examples $\mathcal{D}=\bigcup_{i=1}^{N}\left\{\bar{I}^{(i, j)}\right\}_{j=1}^{M^{(i)}}$, where $M^{(i)}$ is the number of examples from $\bar{I}^{(i)}$. Each $\bar{I}^{(i, j)}=$ $\left\langle\left(s_{1}^{(i, j)}, \gamma_{1}^{(i, j)}, a_{1}^{(i, j)}\right), \ldots,\left(s_{k}^{(i, j)}, \gamma_{k}^{(i, j)}, a_{k}^{(i, j)}\right)\right\rangle$ is a subsequence of tuples in $\bar{I}^{(i)}$, where $a_{1}^{(i, j)}$ is the first action the follower takes after observing the $j$-th instruction in $\bar{I}^{(i)}$, and $a_{k}^{(i, j)}$ is the DONE action completing that instruction. We first estimate the parameters for plan prediction $\theta_{1}$ and action generation $\theta_{2}$ separately (Section 5.1), and then finetune jointly with data augmentation (Section 5.2).

\subsection{Pretraining}

Stage 1: Plan Prediction The input of Stage 1 is the world state $s_{1}$ and the instruction $\bar{x}$ at the head of the queue $\bar{Q} \cdot{ }^{3}$ We generate labels for the four

\footnotetext{
${ }^{3}$ We omit example indices for succinctness.
} 
output distributions using $\bar{I}^{(i, j)}$. The visitation distribution $p\left(\rho \mid s_{1}, \bar{x}\right)$ label is proportional to number of states $s_{t} \in \bar{I}^{(i, j)}$ where the follower is in position $\rho$. The goal and avoidance distributions model how the agent plans to manipulate parts of its environment to achieve the specified goals, but avoid manipulating other parts. In CEREALBAR, this translates to changing the status of cards, or avoiding doing so. For $p\left(\mathrm{GOAL}=1 \mid \rho, s_{1}, \bar{x}\right)$, we set the label to 1 for all $\rho$ that contain a card that the follower changed its selection status in $\bar{I}^{(i, j)}$, and 0 for all other positions. Similarly, for the avoidance distribution $p\left(\right.$ AVOID $\left.=1 \mid \rho, s_{1}, \bar{x}\right)$, the label is 1 for all $\rho$ that have cards that the follower does not change during the interaction $\bar{I}^{(i, j)}$. Finally, for $p$ (NOPASS $\left.=1 \mid \rho, s_{1}, \bar{x}\right)$, the label is 1 for all positions the agent cannot move onto, and zero otherwise. We define four cross-entropy losses: visitation $\mathcal{L}_{V}$, goal $\mathcal{L}_{G}$, avoidance $\mathcal{L}_{A}$, and no passing $\mathcal{L}_{P}$. We also use an auxiliary crossentropy goal-prediction loss $\mathcal{L}_{G^{\prime}}$ using a probability $p_{G}^{\prime}\left(\mathrm{GOAL}=1 \mid \rho, s_{1}, \bar{x}\right)$ we predict from the pre-LINGUNET representation $\mathbf{S}^{\prime}$ by classifying each position. The complete loss is a weighted sum with coefficients: ${ }^{4}$

$$
\begin{aligned}
\mathcal{L}_{1}\left(\theta_{1}\right)= & \lambda_{V} \mathcal{L}_{V}\left(\theta_{1}\right)+\lambda_{G} \mathcal{L}_{G}\left(\theta_{1}\right)+\lambda_{A} \mathcal{L}_{A}\left(\theta_{1}\right) \\
& +\lambda_{P} \mathcal{L}_{P}\left(\theta_{1}\right)+\lambda_{G^{\prime}} \mathcal{L}_{G^{\prime}}\left(\theta_{1}\right) .
\end{aligned}
$$

Stage 2: Action Generation We use the gold distribution to create the input $\mathbf{P}$, and optimize towards the annotated set of actions using teacher forcing (Williams and Zipser, 1989). We compute the loss only over actions taken by the follower:

$$
\mathcal{L}_{2}\left(\theta_{2}\right)=-\sum_{t=1}^{n} \mathbb{1}_{\alpha_{t}=\text { Follower }} p\left(a_{t}\right),
$$

where $p\left(a_{t}\right)$ is computed by Equation 1 .

\subsection{Fine-tuning with Example Aggregation}

Simply combining the separately-trained networks together results in low performance. We perform additional fine-tuning with the two stages combined, and introduce a data augmentation method to learn to recover from error propagation.

Error Propagation Executing a sequence of instructions is susceptible to error propagation, where an agent fails to correctly complete an instruction, and because of it also fails on the following ones. While the collaborative, turn-switching setup allows the leader to adjust their plan following a follower mistake, leaders often strategically issue multiple instructions to use the avail-

\footnotetext{
${ }^{4}$ Additional details are in Appendix E.1.
}

able follower steps optimally. Given an agent failure, subsequent instructions may not align with the state of the world resulting from the follower's error. In supervised learning, we do not have the opportunity to learn to recover from such errors, even when it is relatively simple. This usually requires exploration. However, conventional frameworks like reinforcement learning (RL) or imitation learning (IL) are poorly suitable. In a live interaction, when an agent makes a mistake (e.g., selecting the wrong card), the leader is likely to adjust their actions. Because of this, in a recorded interaction, which contains the leader actions following a correct execution, it is not possible to reliably compute an RL reward for states following erroneous executions. For similar reasons, we cannot compute an IL oracle.

We identify two classes of erroneous states in CerealBar: (a) not selecting the correct set of cards; and (b) finishing with the right card selection, but stopping at the wrong position. ${ }^{5}$ Case (a) requires to modify the model, for example to know when to skip instructions that refer to a state that is no longer possible. We leave this case for future work. We address case (b) by augmenting the data with new examples that are aggregated during learning. Our process is similar to DAGGER (Ross et al., 2011). We alternate between: (a) collecting new training examples using a heuristic oracle, and (b) performing model updates. We generate training examples that demonstrate recovery by starting in an incorrect initial position for an instruction, having arrived there by executing the previous instruction. We train our model to distinguish between the reasoning required for generating implicit actions to correct errors, and explicit actions directly mentioned in the instruction.

Learning with Example Aggregation We alternate between aggregating a new set of recovery examples $\mathcal{D}^{\prime}$ and updating our parameters. At each epoch, we first use the current policy to create new training examples. We run inference for each example $\bar{I}^{(i, j)}$ in $\mathcal{D}$, the original training set, using the current policy. ${ }^{6}$ We compare the state $s^{\prime}$ at the end of execution to the final state in $\bar{I}^{(i, j)}$ to generate an error-recovery example $\bar{I}^{\prime(i, j+1)}$ for the subsequent example $\bar{I}^{(i, j+1)}$. We only generate such examples if the position or rotation of the

\footnotetext{
${ }^{5}$ See Appendix E. 2 for further discussion of the two cases.

${ }^{6} \mathrm{We}$ do not perform inference for the last instruction in an interaction, as there is no subsequent example for which to generate a new example.
} 
agent are different, and there are no other difference between the states. Starting from $s^{\prime}$, we generate the shortest-path sequence of actions that: (a) changes the cards as specified in $\bar{I}^{(i, j+1)}$, and (b) executes DONE in the same position as in $\bar{I}^{(i, j+1)}$. We then create $\bar{I}^{\prime(i, j+1)}$ using $\bar{I}^{(i, j+1)}$ and the new sequence of state-action pairs, and add it to $\mathcal{D}^{\prime}$.

Given the original set of examples $\mathcal{D}$ and the aggregate examples $\mathcal{D}^{\prime}$ we update our model parameters. We randomly sample without replacement at most $\sum_{i=1}^{N} M^{(i)}$ examples, the size of $\mathcal{D}$, from $\mathcal{D}^{\prime}$. We use all the examples in $\mathcal{D}$ and the sampled examples to do a single parameter update epoch. We limit the number of examples from $\mathcal{D}^{\prime}$ to maintain the effect of the original data.

\section{Optimizing with Implicit Action Prediction}

The examples we generate during aggregation often include sequences of state-action pairs that do not align with the instruction, for example when a mentioned spatial relation is incorrect from the new starting position. Such examples require reasoning differently about the text and world state than with the original data. We identify such examples in $\mathcal{D}^{\prime}$ by comparing their follower starting position to the starting position in the original corresponding example in $\mathcal{D}$. If the distance is over two, we treat the examples as requiring implicit actions (Artzi and Zettlemoyer, 2013). All other examples, including all original examples in $\mathcal{D}$ are considered as not requiring implicit reasoning. We encourage the model to reason differently about these examples with a discriminator that classifies if the example requires implicit reasoning or not using the internal activations of LINGUNET.

The discriminator classifies each of the $L$ layers in LINGUNET for implicit reasoning. The goal is to encourage implicit reasoning at all levels of reasoning in the first stage. The probability of implicit reasoning for each LINGUNET layer $l$ is:

$p\left(\right.$ IMPLICT $\left.=1 \mid l, s_{1}, \bar{x}\right)=$

$$
\begin{cases}\sigma\left(\operatorname{AvGPoOL}\left(\mathbf{G}_{1} * \mathbf{K}_{1}^{\mathrm{IMP}}\right)\right) & l=1 \\ \sigma\left(\operatorname{AvGPoOL}\left(\mathbf{H}_{l} * \mathbf{K}_{l}^{\mathrm{IMP}}\right)\right) & l>1\end{cases}
$$

where $\mathbf{K}_{l}^{\mathrm{IMP}}$ are $1 \times 1$ learned kernels and AvgPool does average pooling. We define a cross-entropy loss $\mathcal{L}_{\text {IMP }}$ that averages across the $L$ layers. The complete fine-tuning loss is:

$$
\mathcal{L}\left(\theta_{1}, \theta_{2}\right)=\mathcal{L}_{1}\left(\theta_{1}\right)+\mathcal{L}_{2}\left(\theta_{2}\right)+\lambda_{\mathrm{IMP}} \mathcal{L}_{\mathrm{IMP}}\left(\theta_{1}\right) .
$$

\footnotetext{
${ }^{7}$ Appendix E. 2 describes this process.
}

\section{Cascaded Evaluation}

Sequential instruction scenarios are commonly evaluated using recorded interactions by executing individual instructions or executing complete interactions starting from their beginning (e.g., Chen and Mooney, 2011; Long et al., 2016). Both have limitations. Instruction-level metrics ignore error propagation, and do not accurately reflect the system's performance. In contrast, interaction-level metrics do consider error propagation and capture overall system performance well. However, they poorly utilize the test data, especially when performance is relatively low. When early failures lead to unexpected world states, later instructions become impossible to follow, and measuring performance on them is meaningless. For example, with our best-performing model, $82 \%$ of development instructions become impossible due cascading errors when executing complete interactions.

The two measures may also fail to distinguish models. For example, consider an interaction with three instructions. Two models, A and B, successfully execute the third instruction in isolation, but fail on the two others. They also both fail when executing the entire interaction starting from the beginning. According to common measures, the models are equal. However, if model B can actually recover from failing on the second instruction to successfully execute the third, it means it is better than model A. Both metrics fail to reflect this.

We propose cascaded evaluation, an evaluation protocol for sequential instruction using static corpora. Our method utilizes all instructions during testing, while still accounting for the effect of error propagation. Unlike instruction-level evaluation, cascaded evaluation executes the instructions in sequence. However, instead of starting of starting only from the start state of the first instruction, we create separate examples for starting from the starting state of each instruction in the interaction and continuing until the end of the interaction. For example, given a sequence of three instructions $\langle 1,2,3\rangle$ we will create three examples: $\langle 1,2,3\rangle,\langle 2,3\rangle$, and $\langle 3\rangle$. To evaluate performance in CEREALBAR, we compute two statistics using cascaded evaluation: the proportion of the remaining instructions followed successfully, and the proportion of potential points scored. We only consider the remaining instructions and points left to achieve in the example. For example, for the sequence $\langle 2,3\rangle$, we will subtract any points achieved 
before the second instruction to compute the proportion of potential points scored. Appendix F describes cascaded evaluation formally.

\section{Experimental Setup}

Data We collect 1,202 human-human interactions using Mechanical Turk, split into train (960 games), development (120), and test (122). Appendix $\mathrm{C}$ details data collection and statistics.

Recorded Interactions Metrics We evaluate instruction-level, interaction-level, and cascaded (Section 6) performance. We allow the follower ten steps per turn, and interleave the actions taken by the leader during each turn in the recorded interaction. Instruction execution often crosses turns. At the instruction-level, we evaluate the mean card state accuracy comparing the state of the cards after inference with the correct card state, environment state accuracy comparing both cards and the agent's final position, and action sequence accuracy comparing the generated action sequence with the correct action sequence. For complete interactions, we measure mean full game points. Finally, for cascaded evaluation, we measure the mean proportion of instructions correctly executed and of possible points scored.

Human Evaluation We perform evaluation with human leaders, comparing our model and human followers. Workers are told they will work with a human or an automated follower, but are not told which in each game. We evaluate both human (105 games) and automated agents at the same time (109 games). We evaluate the game scores, and also elicit free-form feedback.

Systems We evaluate three systems: (a) the full model; (b) SEQ2SEQ+ATTN: ${ }^{8}$ sequence-tosequence with attention; and (c) a static oracle that executes the gold sequence of actions in the recorded interaction. We report mean and standard deviation across three trials for development results. We ablate model and learning components, and additionally evaluate the action generator with access to gold plans. ${ }^{9}$ On the test set and for human evaluation, we use the model with the highest proportion of points scored. We provide implementation and learning details in Appendix G.

\section{Results}

Table 1 shows development and test results, including ablations. We consider the proportion of

\footnotetext{
${ }^{8}$ This baseline is similar to Mei et al. (2016).

${ }^{9} \mathrm{We}$ do not measure interaction-level metrics with gold plans as they are only available for the gold start positions.
}

points scored computed with cascaded evaluation as the main metric. Our complete approach significantly outperforms SEQ2SEQ+ATTN. Key to this difference is the added structure within the model and the direct supervision on it. The results also show the large remaining gap to the static oracle. ${ }^{10}$

Our results show how considering error propagation for all available instructions in cascaded evaluation guides different design choices. For example, example aggregation and the implicit discriminator lower performance according to instruction-level metrics, which do not consider error propagation. We see a similar trend for the implicit discriminator when looking at full game points, an interaction-level metric that does not account for performance on over $80 \%$ of the data because of error propagation. In contrast, the proportion of points scored computed using cascaded evaluation shows the benefit of both mechanisms.

Our ablations demonstrate the benefit of each model component. All four distributions help. Without the trajectory distribution (- Trajectory distribution), performance drops almost to the level of SEQ2SEQ+ATTN. This indicates the action predictor is not robust enough to construct a path given only the three other disjoint distributions. While the predicted trajectory distribution contains all information necessary to reach the correct cards and goal location, the other three distributions further improve performance. This is likely because redundancy with the trajectory distribution makes the model more robust to noisy predictions in the trajectory distribution. For example, the GOAL distribution guides the agent to move towards goal cards even if the predicted trajectory is discontinuous. The action generation recurrence is also critical (- Action recurrence), allowing the agent to keep track of which locations it already passed when navigating complex paths that branch, loop, or overlap with themselves.

While we observe that each stage separately performs well after pretraining, combining them without fine-tuning (- Fine-tuning) leads to low performance because of the shift in the second stage input. Providing the gold distributions to the action generator illustrates this (+ Gold plan). Removing early goal auxiliary loss $\mathcal{L}_{G^{\prime}}$ (Section 5.1) leads to a slight drop in performance on all metrics (- Early goal auxiliary). Learning with aggregated recovery examples helps the

\footnotetext{
${ }^{10}$ Appendix F explains the static oracle performance.
} 


\begin{tabular}{|c|c|c|c|c|c|c|}
\hline System & $\begin{array}{c}\text { Card } \\
\text { State Acc. }\end{array}$ & $\begin{array}{c}\text { Env. } \\
\text { State Acc. }\end{array}$ & $\begin{array}{c}\text { Action Seq. } \\
\text { Accuracy }\end{array}$ & $\begin{array}{c}\text { Full Game } \\
\text { Points }\end{array}$ & $\begin{array}{l}\text { Prop. Instr. } \\
\text { Followed }\end{array}$ & $\begin{array}{l}\text { Prop. Points } \\
\text { Scored }\end{array}$ \\
\hline \multicolumn{7}{|c|}{ Development Results \& Ablation Analysis } \\
\hline Full model & $58.2 \pm 0.5$ & $32.6 \pm 0.8$ & $15.8 \pm 0.5$ & $0.66 \pm 0.1$ & $20.5 \pm 1.2$ & $18.1 \pm 0.8$ \\
\hline - Trajectory distribution & $38.5 \pm 2.7$ & $10.1 \pm 2.7$ & $5.5 \pm 2.6$ & $0.29 \pm 0.02$ & $10.0 \pm 0.9$ & $7.9 \pm 0.7$ \\
\hline - GOAL distribution & $56.2 \pm 1.5$ & $30.8 \pm 0.4$ & $14.9 \pm 0.3$ & $0.66 \pm 0.09$ & $17.9 \pm 1.0$ & $15.9 \pm 1.3$ \\
\hline - AVOID distribution & $57.0 \pm 0.3$ & $32.6 \pm 1.6$ & $15.4 \pm 1.3$ & $0.63 \pm 0.04$ & $18.8 \pm 1.5$ & $17.8 \pm 0.7$ \\
\hline - NOPASS distribution & $59.2 \pm 0.5$ & $32.0_{ \pm 0.8}$ & $15.0 \pm 0.5$ & $0.70 \pm 0.03$ & $18.4_{ \pm 0.9}$ & $16.6 \pm 0.9$ \\
\hline - Action recurrence & $42.3 \pm 1.5$ & $16.7 \pm 1.2$ & $10.0_{ \pm 0.7}$ & $0.42 \pm 0.03$ & $12.8 \pm 1.7$ & $10.7 \pm 0.5$ \\
\hline - Fine-tuning & $43.6 \pm 1.9$ & $8.5 \pm 1.1$ & $4.5 \pm 0.5$ & $0.65 \pm 0.09$ & $14.1 \pm 1.3$ & $9.2 \pm 0.9$ \\
\hline - Early goal auxiliary & $57.2 \pm 2.3$ & $31.2 \pm 1.7$ & $14.9_{ \pm 1.6}$ & $0.65 \pm 0.05$ & $17.9 \pm 1.1$ & $16.5 \pm 0.7$ \\
\hline - Example aggregation & $59.4_{ \pm 1.8}$ & $32.0 \pm 1.0$ & $15.7 \pm 0.6$ & $0.65 \pm 0.09$ & $20.4_{ \pm 1.4}$ & $16.5 \pm 0.4$ \\
\hline - Implicit discriminator & $57.5 \pm 2.1$ & $32.7 \pm 1.0$ & $16.4_{ \pm 0.3}$ & $0.70_{ \pm 0.02}$ & $18.8 \pm 1.8$ & $16.7_{ \pm 0.6}$ \\
\hline - Instructions & $15.5 \pm 1.5$ & $2.7 \pm 1.5$ & $1.2 \pm 1.2$ & $0.24 \pm 0.07$ & $4.4 \pm 1.0$ & $4.6 \pm 0.7$ \\
\hline + Gold plan & $87.4 \pm 0.5$ & $80.2 \pm 0.2$ & $63.4 \pm 0.2$ & - & - & - \\
\hline SEQ2SEQ+ATTN & $35.3 \pm 0.8$ & $11.1 \pm 0.5$ & $9.4 \pm 0.5$ & $0.20 \pm 0.04$ & $8.8 \pm 0.1$ & $6.3 \pm 0.1$ \\
\hline Static oracle & 99.7 & 99.7 & 100.0 & 6.58 & 98.5 & 97.9 \\
\hline \multicolumn{7}{|l|}{ Test Results } \\
\hline Full model & 58.4 & 32.1 & 15.6 & 0.62 & 15.4 & 17.9 \\
\hline SEQ2SEQ+ATTN & 37.3 & 10.8 & 8.5 & 0.22 & 8.7 & 6.5 \\
\hline Static oracle & 99.7 & 99.7 & 100.0 & 6.66 & 96.8 & 95.6 \\
\hline
\end{tabular}

Table 1: Development and test results on all systems, including ablation results.

model to learn to recover from errors in previous instructions and increases the proportion of points scored (- Example aggregation). However, without the implicit reasoning discriminator (- Implicit discriminator), the additional examples make learning too difficult, and do not help. Finally, removing the language input (- Instructions) significantly decreases performance, showing that the data is relatively robust to observational biases and language is necessary for the task.

In the human evaluation, we observe a mean of 6.2 points (max of 14) with our follower model, compared to 12.7 (max of 20) with human followers. While this shows there is much room for improvement, it illustrates how human leaders adapt and use the agent effectively. One key strategy of adaptation is to use simplified language that fits the model better. This includes shorter instructions, with 8.5 tokens on average with automated followers compared to 12.3 with humans, and a smaller vocabulary, 578 word types with automated followers and 1037 with humans. In general, human leaders commented that they are able to easily distinguish between automated and human followers, and find working with the automated agent frustrating.

\section{Discussion}

Our human evaluation highlights several directions for future work. While human leaders adapt to the agent, scoring up to 14 points, there remains a significant gap to collaborations with human followers. Reported errors include getting stuck behind objects, selecting unmentioned cards, going in the wrong direction, and ignoring instructions. At least one worker developed a strategy that took advantage of the agent's full observability, writing instructions with only simple card references. An important direction for future work is to remove our full observability assumption. Other future directions include experimenting with using the interaction history, expanding the learning example aggregation to error cases beyond incorrect start positions, and making agent reasoning interpretable to reduce user frustration. CEREALBAR also provides opportunities to study pragmatic reasoning for language understanding (Andreas and Klein, 2016; Fried et al., 2018; Liang et al., 2019). While we currently focus on language understanding by limiting the communication to be unidirectional, bidirectional communication would allow for more natural and efficient collaborations (Potts, 2012; Ilinykh et al., 2019). CerealBar could be easily adapted to allow bidirectional communication, and provide a platform to study challenges in language generation.

\section{Acknowledgments}

This research was supported by the NSF under Grant No. 1750499, an AI2 KSC Award, a Workday Faculty Award, a Google Faculty Award, Unity, and an Amazon Cloud Credits Grant. This material is based on work supported by the National Science Foundation Graduate Research Fellowship under Grant No. DGE-1650441. We thank Valts Blukis, Jin Sun, and Mark Yatskar for comments and suggestions, the workers who participated in our data collection, and the reviewers. 


\section{References}

Anne H. Anderson, Miles Bader, Ellen Gurman Bard, Elizabeth Boyle, Gwyneth Doherty, Simon Garrod, Stephen Isard, Jacqueline Kowtko, Jan McAllister, Jim Miller, Catherine Sotillo, Henry S. Thompson, and Regina Weinert. 1991. The HCRC map task corpus. Language and Speech, 34.

Peter Anderson, Qi Wu, Damien Teney, Jake Bruce, Mark Johnson, Niko Sünderhauf, Ian D. Reid, Stephen Gould, and Anton van den Hengel. 2018. Vision-and-language navigation: Interpreting visually-grounded navigation instructions in real environments. In IEEE Conference on Computer Vision and Pattern Recognition, pages 3674-3683.

Jacob Andreas, Anca Dragan, and Dan Klein. 2017. Translating neuralese. In Proceedings of the Annual Meeting of the Association for Computational Linguistics, pages 232-242.

Jacob Andreas and Dan Klein. 2016. Reasoning about pragmatics with neural listeners and speakers. In Proceedings of the Conference on Empirical Methods in Natural Language Processing, pages 11731182.

Jacob Arkin, Matthew R. Walter, Adrian Boteanu, Michael E. Napoli, Harel Biggie, Hadas KressGazit, and Thomas M. Howard. 2017. Contextual awareness: Understanding monologic natural language instructions for autonomous robots. In IEEE International Symposium on Robot and Human Interactive Communication, pages 502-509.

Yoav Artzi, Dipanjan Das, and Slav Petrov. 2014. Learning compact lexicons for CCG semantic parsing. In Proceedings of the Conference on Empirical Methods in Natural Language Processing, pages 1273-1283.

Yoav Artzi and Luke Zettlemoyer. 2011. Bootstrapping semantic parsers from conversations. In Proceedings of the Conference on Empirical Methods in Natural Language Processing, pages 421-432.

Yoav Artzi and Luke Zettlemoyer. 2013. Weakly supervised learning of semantic parsers for mapping instructions to actions. Transactions of the Association of Computational Linguistics, 1:49-62.

Valts Blukis, Nataly Brukhim, Andrew Bennett, Ross A. Knepper, and Yoav Artzi. 2018a. Following high-level navigation instructions on a simulated quadcopter with imitation learning. In Proceedings of the Robotics: Science and Systems Conference.

Valts Blukis, Dipendra Misra, Ross A. Knepper, and Yoav Artzi. 2018b. Mapping navigation instructions to continuous control actions with position visitation prediction. In Proceedings of the Conference on Robot Learning.
Alexander Broad, Jacob Arkin, Nathan Ratliff, Thomas Howard, and Brenna Argall. 2017. Real-time natural language corrections for assistive robotic manipulators. The International Journal of Robotics Research, 36(5-7):684-698.

David L. Chen and Raymond J. Mooney. 2011. Learning to interpret natural language navigation instructions from observations. In Proceedings of the $\mathrm{Na}$ tional Conference on Artificial Intelligence.

Howard Chen, Alane Suhr, Dipendra Misra, Noah Snavely, and Yoav Artzi. 2019. Touchdown: Natural language navigation and spatial reasoning in visual street environments. In IEEE Conference on Computer Vision and Pattern Recognition.

Alex Djalali, David Clausen, Sven Lauer, Karl Schultz, and Christopher Potts. 2011. Modeling expert effects and common ground using questions under discussion. In AAAI Fall Symposium: Building Representations of Common Ground with Intelligent Agents.

Alex Djalali, Sven Lauer, and Christopher Potts. 2012. Corpus evidence for preference-driven interpretation. In Logic, Language and Meaning, pages 150159.

Felix Duvallet, Thomas Kollar, and Anthony Stentz. 2013. Imitation learning for natural language direction following through unknown environments. In IEEE International Conference on Robotics and Automation, pages 1047-1053.

Jeffrey L. Elman. 1990. Finding structure in time. Cognitive Science, 14:179-211.

Katrina Evtimova, Andrew Drozdov, Douwe Kiela, and Kyunghyun Cho. 2017. Emergent communication in a multi-modal, multi-step referential game. In Proceedings of the International Conference on Learning Representations.

Daniel Fried, Jacob Andreas, and Dan Klein. 2018. Unified pragmatic models for generating and following instructions. In Proceedings of the Conference of the North American Chapter of the Association for Computational Linguistics: Human Language Technologies, pages 1951-1963.

He He, Anusha Balakrishnan, Mihail Eric, and Percy Liang. 2017. Learning symmetric collaborative dialogue agents with dynamic knowledge graph embeddings. In Proceedings of the Annual Meeting of the Association for Computational Linguistics, pages 1766-1776.

He He, Derek Chen, Anusha Balakrishnan, and Percy Liang. 2018. Decoupling strategy and generation in negotiation dialogues. In Proceedings of the Conference on Empirical Methods in Natural Language Processing, pages 2333-2343.

Sepp Hochreiter and Jürgen Schmidhuber. 1997. Long short-term memory. Neural computation, 9. 
Hengyuan $\mathrm{Hu}$, Denis Yarats, Qucheng Gong, Yuandong Tian, and Mike Lewis. 2019. Hierarchical decision making by generating and following natural language instructions. CoRR, abs/906.00744.

Nikolai Ilinykh, Sina Zarrieß, and David Schlangen. 2019. MeetUp! A corpus of joint activity dialogues in a visual environment. CoRR, abs/1907.05084.

Mohit Iyyer, Wen-tau Yih, and Ming-Wei Chang. 2017. Search-based neural structured learning for sequential question answering. In Proceedings of the Annual Meeting of the Association for Computational Linguistics, pages 1821-1831.

Jin-Hwa Kim, Nikita Kitaev, Xinlei Chen, Marcus Rohrbach, Yuandong Tian, Dhruv Batra, and Devi Parikh. 2019. CoDraw: Collaborative Drawing as a Testbed for Grounded Goal-driven Communication. CoRR, abs/1704.04517.

Theodora Koulouri and Stanislao Lauria. 2009. Exploring miscommunication and collaborative behaviour in human-robot interaction. In Proceedings of the SIGDIAL Conference, pages 111-119.

Mike Lewis, Denis Yarats, Yann Dauphin, Devi Parikh, and Dhruv Batra. 2017. Deal or no deal? End-to-end learning of negotiation dialogues. In Proceedings of the Conference on Empirical Methods in Natural Language Processing, pages 2443-2453.

Claire Liang, Julia Proft, Erik Andersen, and Ross A. Knepper. 2019. Implicit communication of actionable information in human-AI teams. In Proceedings of the CHI Conference on Human Factors in Computing Systems, pages 95:1-95:13.

Karen E. Lochbaum. 1998. A collaborative planning model of intentional structure. Computational Linguistics, 24(4):525-572.

Reginald Long, Panupong Pasupat, and Percy Liang. 2016. Simpler context-dependent logical forms via model projections. In Proceedings of the Annual Meeting of the Association for Computational Linguistics, pages 1456-1465.

Matthew MacMahon, Brian Stankiewics, and Benjamin Kuipers. 2006. Walk the talk: Connecting language, knowledge, action in route instructions. In Proceedings of the National Conference on Artificial Intelligence.

Hongyuan Mei, Mohit Bansal, and Matthew R. Walter. 2016. Listen, attend, and walk: Neural mapping of navigational instructions to action sequences. In Proceedings of the AAAI Conference on Artificial Intelligence.

Scott Miller, David Stallard, Robert Bobrow, and Richard Schwartz. 1996. A fully statistical approach to natural language interfaces. In Proceedings of the Annual Meeting of the Association for Computational Linguistics, pages 55-61.
Dipendra Misra, Andrew Bennett, Valts Blukis, Eyvind Niklasson, Max Shatkhin, and Yoav Artzi. 2018. Mapping instructions to actions in 3D environments with visual goal prediction. In Proceedings of the Conference on Empirical Methods in Natural Language Processing, pages 2667-2678.

Dipendra Misra, John Langford, and Yoav Artzi. 2017. Mapping instructions and visual observations to actions with reinforcement learning. In Proceedings of the Conference on Empirical Methods in Natural Language Processing, pages 1004-1015.

Christopher Potts. 2012. Goal-driven answers in the Cards dialogue corpus. In Proceedings of the West Coast Conference on Formal Linguistics, pages 120.

Stéphane Ross, Geoffrey Gordon, and Drew Bagnell. 2011. A reduction of imitation learning and structured prediction to no-regret online learning. In Proceedings of the International Conference on Artificial Intelligence and Statistics.

Candace L. Sidner, Carolyn Boettner, and Charles Rich. 2000. Lessons learned in building spoken language collaborative interface agents. In $A N L P$ NAACL Workshop: Conversational Systems.

Alane Suhr and Yoav Artzi. 2018. Situated mapping of sequential instructions to actions with single-step reward observation. In Proceedings of the Annual Meeting of the Association for Computational Linguistics, pages 2072-2082.

Alane Suhr, Srinivasan Iyer, and Yoav Artzi. 2018. Learning to map context-dependent sentences to executable formal queries. In Proceedings of the Conference of the North American Chapter of the Association for Computational Linguistics: Human Language Technologies, pages 2238-2249.

Stephanie Tellex, Thomas Kollar, Steven Dickerson, Matthew R. Walter, Ashis Gopal Banerjee, Seth Teller, and Nicholas Roy. 2011. Understanding natural language commands for robotic navigation and mobile manipulation. In Proceedings of the $\mathrm{Na}$ tional Conference on Artificial Intelligence.

Jesse Thomason, Shiqi Zhang, Raymond Mooney, and Peter Stone. 2015. Learning to interpret natural language commands through human-robot dialog. In Proceedings of the International Joint Conference on Artificial Intelligence.

Takuma Udagawa and Akiko Aizawa. 2019. A natural language corpus of common grounding under continuous and partially-observable context. In Proceedings of the Conference on Artificial Intelligence.

Dmitry Ulyanov, Andrea Vedaldi, and Victor S. Lempitsky. 2017. Improved texture networks: Maximizing quality and diversity in feed-forward stylization and texture synthesis. In IEEE Conference on Computer Vision and Pattern Recognition, pages 41054113. 
Andreas Vlachos and Stephen Clark. 2014. A new corpus and imitation learning framework for contextdependent semantic parsing. Transactions of the Association for Computational Linguistics, 2:547-560.

Harm de Vries, Kurt Shuster, Dhruv Batra, Devi Parikh, Jason Weston, and Douwe Kiela. 2018. Talk the Walk: Navigating New York City through grounded dialogue. arXiv preprint arXiv:1807.03367.

Sida I. Wang, Percy Liang, and Christopher D. Manning. 2016. Learning language games through interaction. In Proceedings of the Annual Meeting of the Association for Computational Linguistics, pages 2368-2378.

Jason Williams, Antoine Raux, Deepak Ramachandran, and Alan Black. 2013. The dialog state tracking challenge. In Proceedings of the SIGDIAL Conference, pages 404-413.

Ronald J. Williams and David Zipser. 1989. A learning algorithm for continually running fully recurrent neural networks. Neural Computation, 1:270-280. 